\title{
Influence of Oxygen at Atmospheric and High Pressure on the Developing Tooth Germ in Rat Embryos
}

\author{
TETSUO NAKAMOTO and JOHN W. BEAN \\ Department of Physiology, Medical School and Department of \\ Oral Biology, School of Dentistry, University of Michigan, \\ Ann Arbor, Michigan 48104, USA
}

Repeated exposure of pregnant rats to oxygen at high pressure $(65$ psi) twice a day for seven days increased the height of the tooth germ and ameloblastic layer of the embryo, but the width and odontoblastic layer were not changed. Exposure of pregnant rats to oxygen at atmospheric pressure stimulated the body growth of the embryo in utero.

It is well-known that if animals are exposed repeatedly to oxygen at high pressure with attendant oxygen convulsion, residual motor disability in fore and hind limbs may occur. ${ }^{1}$ Oxygen at high pressure also induces pulmonary damage, ${ }^{2,3}$ convulsive seizure, ${ }^{4}$ and changes in the endocrine system. ${ }^{5}$ Earlier experiments have indicated that increased oxygen tension might cause an increase in body weight. ${ }^{6,7}$ Nakamoto and Bean $^{6}$ have found that oxygen at high pressure accelerated the growth rate of incisors in young rats, but skeletal bone growth and body weight were decreased compared with that in the rats exposed to air at atmospheric pressure and oxygen at atmospheric pressure. At the present time, there is no information available as to whether oxygen at atmospheric pressure or at high pressure alters embryonic tooth growth in utero. Specifically, it is of interest to determine whether exposure of the pregnant rat to oxygen at atmospheric pressure or oxygen at high pressure causes an accelerated growth resulting in an increase in size of the tooth germ of the fetus. Experiments were carried out to determine whether pure

This investigation was supported, in part, by Grant H.E. 01646, USPHS and Grant DE-00013 from the National Institutes of Health, Bethesda, Md.

Received for publication July 2, 1971. oxygen or oxygen at high pressure affects embryonic growth or growth of tooth germ, or modifies the two germinal layers.

\section{Materials and Methods}

In these studies, three experimental environments were used: air at atmospheric pressure (AAP), oxygen at atmospheric pressure (OAP) and oxygen at high pressure (OHP). For OAP exposures, a special glass-walled chamber ( 12 by 24 by 12 inches) was used. A copper coil for circulation of fluid to regulate temperature was suspended from the cover. Oxygen was supplied from commercial cylinders connected to inflow and outflow tubes at opposite ends of the chamber through which a small continuous flow of oxygen was maintained throughout exposures (Fig 1).

The exposures to OHP were made in two cylindrical pressure chambers 9 and 11 inches in diameter and 20 inches long, equipped with pressure gauge, light, glass viewing port, and screen floor under which soda lime was placed for the absorption of carbon dioxide. The two chambers were connected to an oxygen supply tank by a $\mathrm{Y}$ tube (Fig 2). The thermometer inside the chamber recorded the temperature, which was maintained at about $27 \mathrm{C}$. Carbon dioxide analysis of chamber gas with a gas analyzer* indicated that the content was less than $0.1 \%$. An oxygen analyzer $f$ was used to ensure a continuous high concentration of oxygen. Seven Sprague-Dawey male and 14 female rats were used. The rats were provided with the same laboratory diet and water ad libitum throughout the experimen-

\footnotetext{
* Model LB-1, Beckman Instrument, Inc., Spinco Div., Palo Alto, Calif.

† Model E2, Fullerton, Calif.
} 


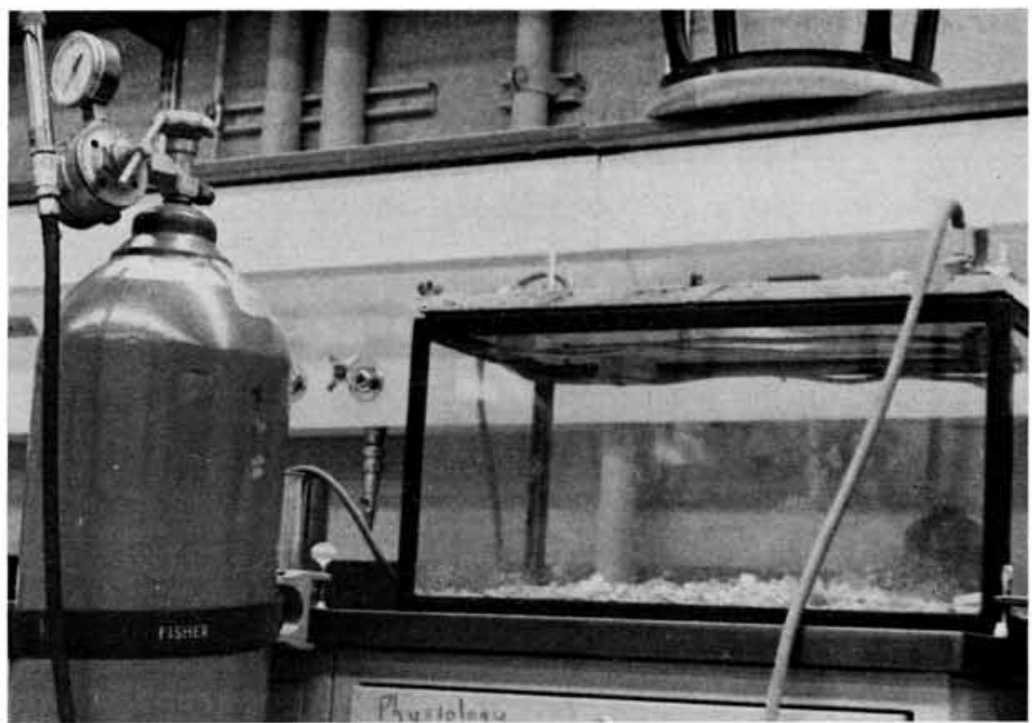

FIG 1.-Special glass-walled chamber through which a continuous stream of oxygen was directed. Rat was exposed to OAP for about 37 minutes.

tal periods. One male and two female rats were put together in a cage and every 12 hours a vaginal smear was taken to determine the time of fertilization as closely as possible. The day on which sperm appeared on the smear was taken as a first day of fertilization, and immediately the female was separated from the male.

Ten pregnant female rats were separated at random as follows: three for AAP exposure, three for OAP exposure, and four for OHP exposure. Repeated exposures

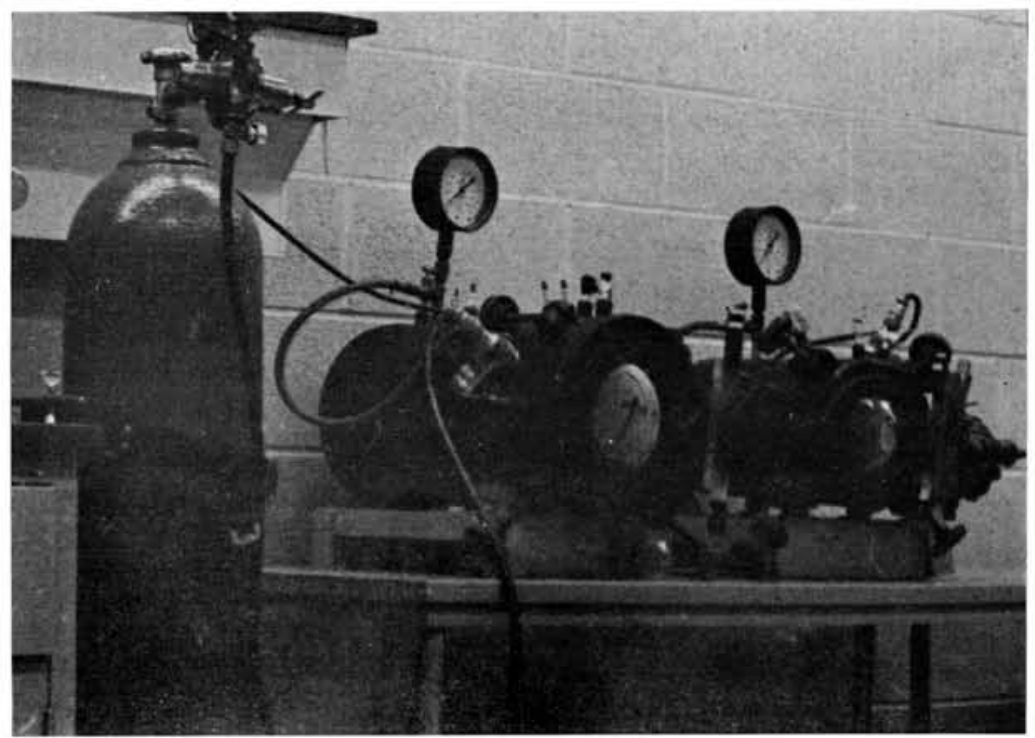

FIg 2.-Two high-pressure chambers connected with Y tube from oxygen supply cylinder. Soda lime was placed below a screen floor to absorb the excess carbon dioxide. 
were begun on the 15 th day after fertilization. Before each exposure, the chambers were washed free of air by a rapid flow of oxygen. The duration of exposure to OHP was determined by the individual responses of the rats, as indicated by the onset of convulsive seizure; after this stage decompression was carried out to atmospheric pressure. The mean compression time, pressurized time, and decompression time were about $3.5,12.5$, and 21 minutes, respectively. In the first group, four pregnant rats were exposed to OHP at 65 psi 13 times over a period of seven days. The second group was exposed to $\mathrm{OAP}$ in a program identical to that of the OHP group.

The average oxygen exposure for the OAP group was about 37 minutes, the same as that of the OHP exposures. During this exposure period, the food of the AAP-exposed group was removed from the cages to ensure equal feeding time. If the rats did not deliver offspring in a normal gestation period of 22 days, they were anesthetized 12 hours later with sodium pentobarbital and the fetuses were removed surgically. Those that were alive were weighed immediately, and those that had died in utero were counted and discarded.

Thirty offspring, ten from each AAP, OAP, and OHP group, were used for measuring tooth germ growth. These were selected randomly as follows: three fetuses were taken from each of two mothers, and four were taken from the third. The left and right sides of the mandibles were removed under the dissection microscope, fixed with Bouin's solution, and embedded in paraffin. Sagittal sections 10 micrometers $(\mu \mathrm{m})$ thick were made of the mandibular structures from the buccal to the lingual aspect. Every tenth section was placed on a glass slide and stained with hematoxylin and eosin. From these sections, the size of the tooth germ of the first molar and the size of the cell layers were measured as previously described. The reproducibility of these measurements was assured by the method described in the earlier paper. ${ }^{8}$ The tooth germ and its mandible were examined microscopically to detect any changes that might have occurred. The data were analyzed using analysis of variance ( $F$ test) and Scheffe's test for multiple comparison.
Differences at the $5 \%$ level were considered statistically significant.

\section{Results}

The data obtained from male and female offspring were pooled because statistical analysis showed no significant difference attributable to sex $(P>0.05)$. Four adult female rats did not become pregnant. One pregnant rat exposed to OHP died during exposure. At the 22nd day of pregnancy, two rats exposed to OAP and two rats exposed to OHP failed to deliver offspring. They were killed 12 hours later. Four offspring of rats exposed to OAP and five of rats exposed to OHP did not move at the time of removal from the uterus, therefore, they were assumed to have died in utero.

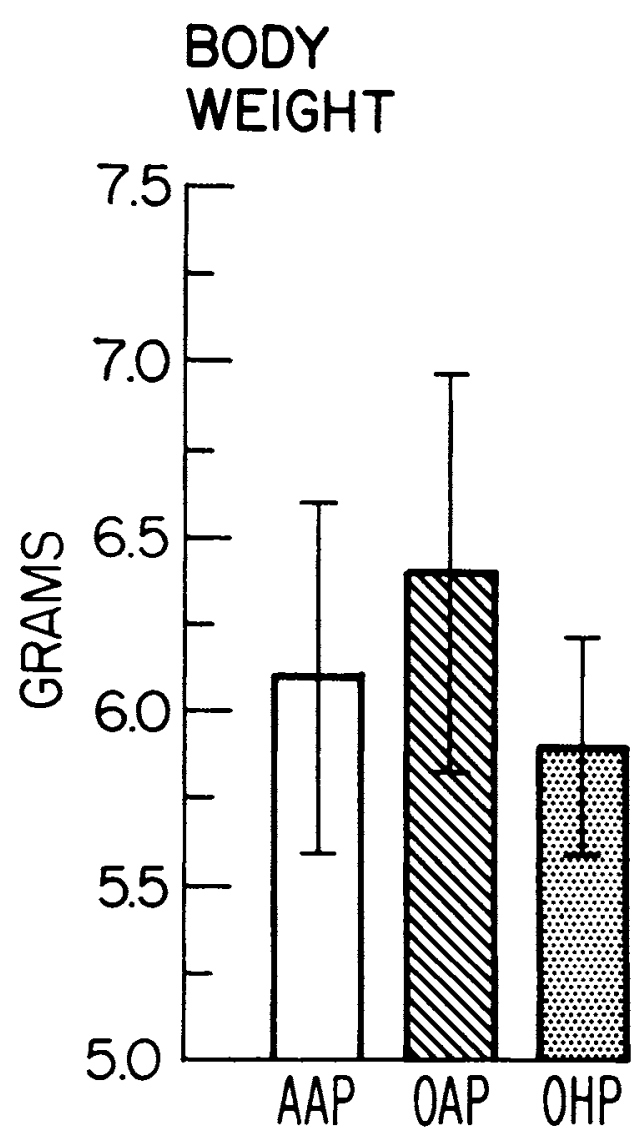

FIG 3.-Body weight of all the fetuses at 22 days plus 12 hours. Rats that were not delivered normally at 22 days were removed by cesarean section. Each bar represents the average of 25 to 30 offspring. 
All pregnant rats exposed to AAP delivered their offspring at 22 days or within 12 hours thereafter. However, three offspring from rats exposed to AAP were stillborn. The total number of offspring from each group was 30 from the group exposed to AAP, 35 from those exposed to OAP, and 32 from those exposed to OHP. None of the mothers showed any particular adverse effects such as sudden decrease in weight or any pathologic appearance during the experimental periods of either OAP or OHP exposure. At the time of delivery, either naturally or surgically, the offspring from rats exposed to OAP were heavier than either those from rats exposed to AAP $(P<0.05)$ or to OHP $(P<0.01)$ (Fig 3). However, offspring from rats exposed to AAP or OHP showed no significant difference $(P>0.05)$ (Fig 3$)$.

The width of the tooth germ was not significantly different in any of these groups
$(P>0.05)$ (Fig 4). However, the height of the tooth germ in offspring from rats exposed to OHP was statistically greater than that of offspring from rats exposed to AAP $(P<0.01)$. Offspring from rats exposed to AAP or OAP were not significantly different $(P>0.05)$ (Fig 4$)$. There was no measurable dentin formed at the time of delivery. The ameloblastic layer of the offspring from rats exposed to AAP was not significantly different from that of offspring from rats exposed to OAP $(P>0.05)$, but that of the offspring from rats exposed to OHP was greater statistically than that of offspring from OAP-exposed rats $(P<0.01)$ (Fig 5). The odontoblastic layer of the offspring from rats exposed to OAP and OHP also was greater than that of the offspring from AAP-exposed rats, but these differences were not statistically significant $(P>0.05)$ (Fig 5). Sections of the tooth germ and the

\section{WIDTH}

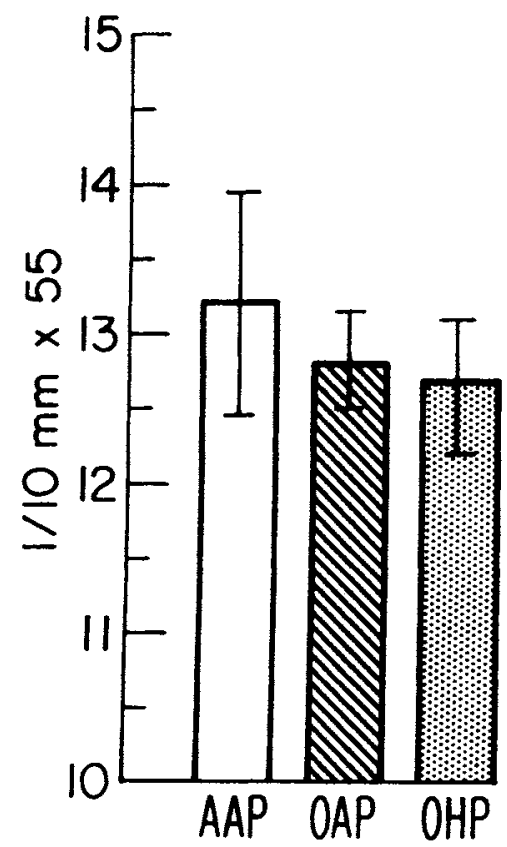

\section{HEIGHT}

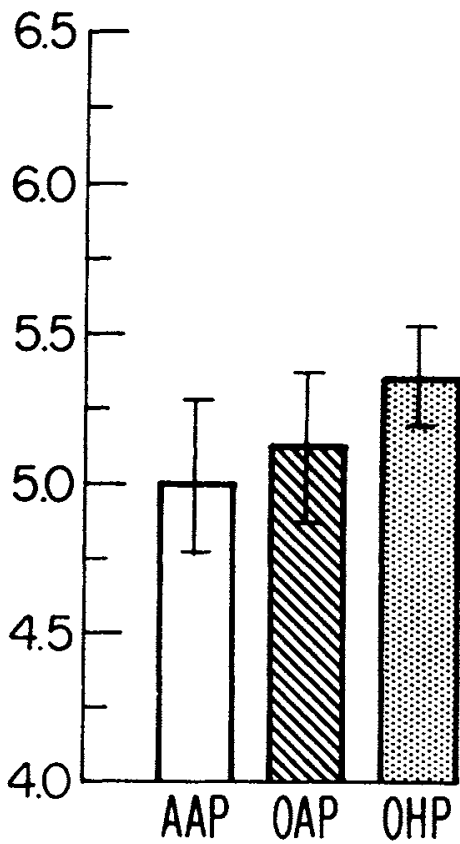

FIg 4.-Comparison of the width and height of the tooth germ of the first molar of offspring from rats exposed to AAP, OAP, and OHP. Number on the vertical scales is arbitrary, based on the photographic enlargement factor. Actual size of tooth germ at 10 in the vertical scale is 0.0182 $\mathrm{mm}$. Each bar represents the average of 55 to 65 measurements. 

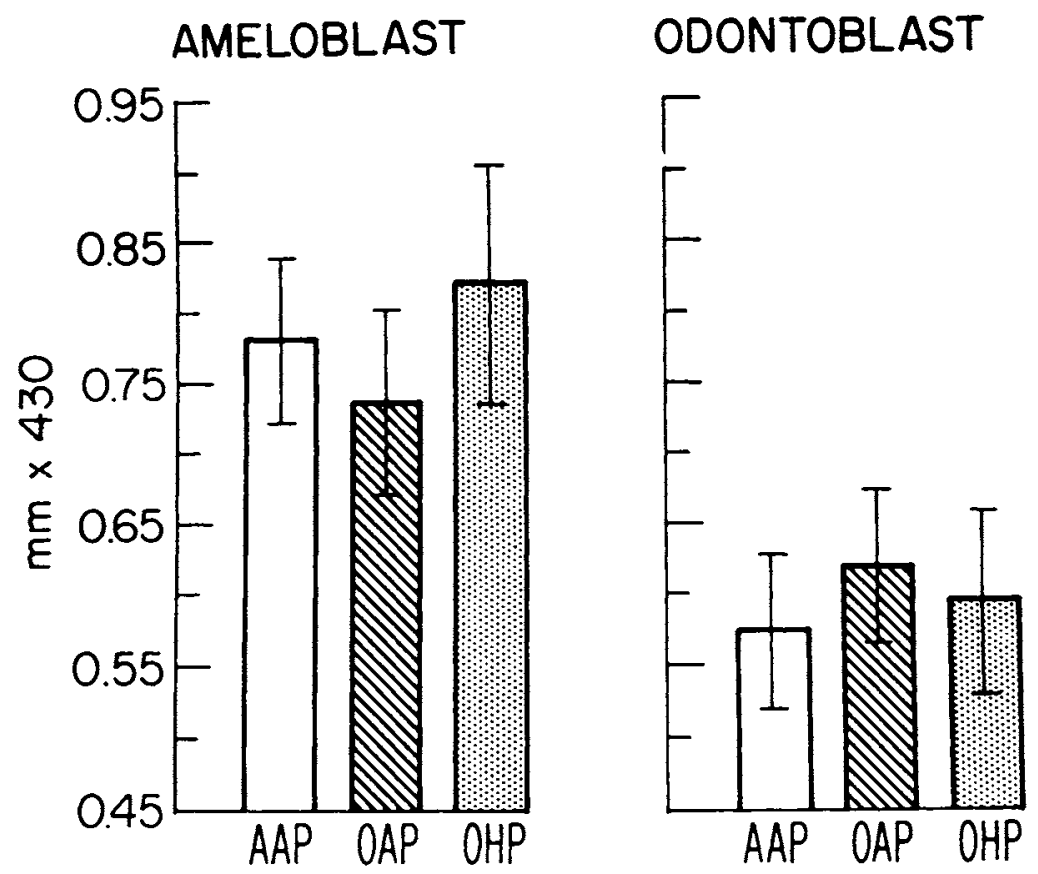

FIG 5.-Comparison of the thickness of the ameloblastic and the odontoblastic layer of the tooth germ of offspring from rats exposed to AAP, OAP, and OHP. Measurements were made with an ocular micrometer at a magnification $\times 430$. Each bar represents average of 55 to 65 measurements.

body of the mandible showed no pathologic changes in the offspring from rats exposed to OAP or OHP.

\section{Discussion}

The authors ${ }^{6}$ previously reported that intermittent exposure to pure oxygen at atmospheric pressure might stimulate body growth (body weight), but the adverse effect of oxygen exposures eventually becomes evident, as indicated by some loss in the later stages of experiment. The present study also showed that OAP exposure of pregnant rats for a short period resulted in a slight increased body weight as compared with AAP and OHP exposure. Evidence showed that this increased weight was due to the increased weight of the fetus (Fig 3). However, one might speculate that some increased weight might also have come from the pregnant rat itself; although in the present experiments it was impossible to determine accurately the comparative weights of the mother rats after delivery, because two thirds of the deliveries were done by opening the uterus in the groups exposed to OAP or OHP.

With OHP exposure, there was no decrease in body weight in pregnant rats as had been observed in early exposures of nonpregnant adult rats. ${ }^{6}$ One might speculate that these pregnant rats exposed to OHP have a greater resistance in pregnancy to the temporarily debilitating effect (oxygen toxicity).

In regard to the OAP exposures, our results showed that the weight of the offspring from rats exposed to OAP was greater than that of the controls (Fig 3). This increase of body weight might be because of a better oxygen supply to the embryo through the umbilical vein; it has been shown that environmental oxygen affects the fetus, ${ }^{9}$ and because of the shape of the oxygen dissociation hemoglobin curve, a relatively small increase in oxygen tension of the umbilical 
vein blood results in a relatively large increase in the oxygen saturation. ${ }^{10}$

Early evidence has shown that the initiation of the dental lamina of the first molar occurred at the 13th day in utero. ${ }^{11}$ In our experiments, the first exposure to oxygen was done on the 15 th day of pregnancy because of the possibility that earlier exposures might induce miscarriage and because at the 15 th day the dental lamina was still primitive. The present data showed that the width of the tooth germ was not increased by exposure to OHP $(P>0.05)$; however, the height was greater in offspring from rats exposed to OHP than in offspring from rats exposed to AAP (Fig 4). This increased growth (height) is essentially the same as that of the adult rats under the same conditions. $^{6}$ Thus, the data show that OHP exposure increases growth of the tooth germ as well as that of the adult incisors. The explanation for this greater rate of growth is not evident. The growth rate of the tooth has been shown to be closely related to pituitary function. ${ }^{12,13}$ With exposure to OHP, there is involvement of the endocrine system, particularly the hypophyseal sympathoendocrine system. ${ }^{14}$ Past evidence ${ }^{15-17}$ showed that destruction of the sympathetic nerve fibers resulted in an increased growth rate of the teeth, possibly because of vasodilatation. If this is so, one might expect that hyperactivity of the hypophyseal sympathoendocrine system that occurs after OHP exposure would result in decreased tooth germ growth because of vasoconstriction.

However, previous ${ }^{6}$ and present evidence shows that such severe exposure resulted in increased tooth growth. Present experimental data indicate that exposures of the pregnant rat to OHP stimulate the odontogenic epithelium. ${ }^{11}$ From these data, one may reasonably speculate that the greater tooth germ growth in the fetus of the rat exposed to OHP to the point of precipitation of oxygen convulsions might be due to an increased release of the growth hormone or some unknown substances from the mother.

Other investigators have found that the development of chick and frog embryos was accelerated in some stages with moderate increases in oxygen tension. ${ }^{18}$ However, in our experiment, it was found that oxygen at atmospheric pressure did not affect tooth germ growth (Fig 4). The thickness of the odontoblastic layer was the same in the offspring from rats exposed to OAP or OHP as that of the controls. However, the thickness of the ameloblastic layer in the offspring from rats exposed to OAP was decreased as compared with that in offspring from rats exposed to OHP.

This raises the question as to whether ameloblast cells are more sensitive to oxygen toxicity than the cells of the odontoblast. If this were so, one might expect that the thickness of the ameloblastic layer in the offspring from rats exposed to OHP would also be decreased. However, this was not true. In fact, the ameloblastic layer was increased in the offspring from rats exposed to OHP as compared with that in offspring from rats exposed to OAP (Fig 5). Further investigation might clarify these points. In our experiment, histological studies did not show any pathologic changes such as hyperemia or hemorrhage inside the tooth germ as was observed in adult dogs by others using low barometric pressure. ${ }^{19}$ This difference in reaction may be related to the pregnancy. It also is of interest that in each group of rats exposed to OAP and OHP, two of three did not deliver offspring at the normal time of gestation. This suggests that this type of oxygen exposure has some adverse effects on the duration of the gestational period.

\section{Conclusions}

Pregnant rats were exposed to OHP (65 psi) for a period of seven days beginning at the 15th day after conception as determined by vaginal smear. These exposures were carried out intermittently, twice a day, to the point of convulsion; after this, decompression was carried out in stages to prevent excessive lung damage or death of the rats. There was a slight decrease in body weight in the newborn rats (22 days), but no significant growth in the width of the tooth germ was noted. However, the height of the tooth germ was increased over that of the controls in the offspring from rats that were exposed to AAP. The thickness of the odontoblastic layer was not altered, but that of the ameloblastic layer was increased. Exposure of the mother to OHP increased elongation of the tooth germ in the embryo in utero. 
The body weight of the offspring from rats exposed to OAP (exposure was concurrent with OHP exposure) was greater than that of offspring from rats exposed to AAP or OHP. The width and height of the tooth germ were not significantly altered, and the thickness of the ameloblastic and odontoblastic layers was unchanged.

The authors thank J. K. Avery, professor of oral biology, for his suggestions, and A. K. Saiki, professor of pathology, University of North Dakota, for his microscopic examinations.

\section{References}

1. Bean, J.W., and Siegrried, E.C.: Transient and Permanent After-effects of Exposure to Oxygen at High Pressure, Am J Physial 143:656-665, 1945.

2. VanDen Brenk, H.A.S., and JAMieson, D.: Pulmonary Damage Due to High Pressure $\mathrm{O}_{2}$ Breathing in Rats, Aust $J$ Exp Biol Med Sci 40:37-50, 1962.

3. BEAN, J.W.: Effects of Oxygen at Increased Pressure, Physiol Rev 25:1-147, 1945.

4. Thomson, W.A.R.: The Physiology of Deep Sea Diving, $B r$ Med $J$ 2:208-210, 1935.

5. Bean, J.W., and Johnson, P.C.: Adrenocortical Response to Single and Repeated Exposure to Oxygen at High Pressure, Am J Physiol 179:410-414, 1954.

6. Nakamoto, T., and Bean, J.W.: Differences in Growth Pattern of Bone and Incisor of Rats Exposed to $\mathrm{O}_{2}$ at Atmospheric and High Pressure, Arch Oral Biol 17:799-810, 1972.

7. Dines, J.H., and Hiatt, E.P.: Prolonged Exposure of Young Rats to an Oxygen Atmosphere at Reduced Pressure, $J$ Appl Physiol 19:17-20, 1964.

8. NAKamoto, T., and Wilson, R.H.: The Growth Rate of Tooth Germ in Newlyborn Rats Injected with Cortisone Acetate, Arch Oral Biol 13:1107-1118, 1968.
9. Dawes, G.S.: Oxygen in the Fetus, in DICKENS, F., and NEI, E. (eds):Oxygen in the Animal Organism, London: Pergamon Press, 1964, pp 559-568.

10. Parker, H.R., and Purves, M.J.: Some Effects of Maternal Hyperoxia and $\mathrm{Hy}$ poxia on the Blood Gas Tensions and Vascular Pressure in the Foetal Sheep, $Q J$ Exp Physiol 52:205-221, 1967.

11. Schour, I., and Massler, M.: The Teeth, in FARRIS, E.J., and GRIFFITH, J.Q. (eds): The Rat in Laboratory Investigation, New York: Hafner Publishing Co., 1962, pp 3, $106,112$.

12. Nakamoto, T., and Wilson, R.H.: Effect of Combinations of Temperature and Light on Skeletal Development and the Growth Rate of Incisors in Rats, $J$ Dent Res 48: 461-466, 1969.

13. Schour, I., and VAN Dyke, H.B.: Changes in the Tooth Following Hypophysectomy. I. Changes in the Incisors of the White Rat, Am J Anat 50:397-433, 1932.

14. BEAN, J.W.: The Hypophysis as a Determinant in the Reaction of the Mammal to Oxygen at High Pressure, Am J Physiol 170:508-517, 1952.

15. LeIST, M.: Uber den einfluss des vegetativen nervensystems auf die zahne, $Z$ Stomatol 25:765-776, 1927.

16. KING, J.D.: Dietary Deficiency, Nerve Lesions and the Dental Tissue, $J$ Physiol 88: 62-76, 1937.

17. Edwards, L.F., and Kitchin, P.C.: Does Resection of the Nerves Supplying the Mandible Affect Tooth Development? $J$ Dent Res 17:115-124, 1938.

18. Nelsen, O.E.: Increased Partial Pressure of $\mathrm{O}_{2}$ and the Acceleration of Development in Early Chick and Frog Embryo: Significance of Early Primitive Streak Activities, Growth 22:189-213, 1958.

19. ORBAN, B.; RITCHEY, B.; and ZANDER, H.A.: Experimental Study of Puip Changes Produced in the Decompression Chamber, $J$ Dent Res 25:299-309, 1946. 